\title{
Considerations on the Selection of Operating Modes for Future Coaxial-Cavity Gyrotrons for DEMO
}

\author{
T. Ruess ${ }^{1}$, K. A. Avramidis ${ }^{1}$, G. Gantenbein ${ }^{1}$, S. Illy ${ }^{1}$, Z. Ioannidis ${ }^{1}$, P. Kalaria $^{1}$, M. Obermaier ${ }^{1}$, \\ I. Gr. Pagonakis ${ }^{1}$, S. Ruess ${ }^{1,2}$ T. Rzesnicki ${ }^{1}$, M. Thumm ${ }^{1,2}$ and J. Jelonnek ${ }^{1,2}$ \\ ${ }^{1} \mathrm{IHM},{ }^{2} \mathrm{IHE}$, Karlsruhe Institute of Technology (KIT), Kaiserstr. 12, 76131 Karlsruhe, Germany
}

\begin{abstract}
At KIT, a modular 170 GHz, 2 MW TE $34,19-$ mode coaxial-cavity gyrotron with advanced water cooling is ready for tests. The successful operation of this tube will be a first important step towards a possible future DEMO gyrotron. Nevertheless, looking forward, there are two questions to be answered: (i) what potential does the existing coaxial cavity offer with regards to MW-class multi-frequency operation also at higher frequencies, and (ii) what could be a different mode selection to achieve an even higher output power in a more compact gyrotron design. To provide an answer to (i), based on the $170 \mathrm{GHz}, 2 \mathrm{MW}$ preprototype the multi-frequency operation at multiples of the resonance frequency of the diamond disc RF output window was carried out. Additionally, a slightly modified cavity design was introduced. To answer the question (ii), the $T_{25,22}$-mode was chosen and compared with the results got for the TE34,19-mode. The extreme volume $\mathbf{T E}_{25,22}$-mode allows to reduce the beam radius by around $25 \%$ and to increase the RF output power of the gyrotron by up to $30 \%$.
\end{abstract}

\section{INTRODUCTION}

Gyrotrons are the only microwave sources producing a megawatt-level RF output power in the sub-THz frequency range. Therefore, gyrotrons are the only option to be used for efficient electron cyclotron resonance heating and electron cyclotron current drive (ECRH\&CD) in nuclear fusion devices. For example, at the stellarator $\mathrm{W} 7-\mathrm{X}$, fusion gyrotrons operating at $140 \mathrm{GHz}$ and at $1 \mathrm{MW}(1800 \mathrm{~s})$ have already demonstrated their excellent reliable performance [1]. In Europe, the major fusion power plants under development are ITER at Cadarache [2] and EU DEMO (Demonstration Power Plant) [3]. The EU DEMO, planned as next step after ITER is under development within the Horizon2020 EUROfusion program.

The first version of the EU ITER gyrotron will use the conventional hollow-cavity technology. The operating mode was chosen to be the $\mathrm{TE}_{32,9}$-mode [4,5]. An issue of the hollowcavity technology is the increasing mode competition with increasing operating mode order. Operation in high order modes is necessary when higher power and frequency is targeted. Coaxial-type cavities including a corrugated inner conductor reduce mode competition, and, additionally, the voltage depression caused by the space charge of the electron beam [6,7]. Hence, the coaxial technology is preferred in EU for multi-MW fusion gyrotrons. Experimental results of the KIT modular coaxial-cavity pre-prototype gyrotron show an output power of above $2 \mathrm{MW}$ at $170 \mathrm{GHz}$ in short-pulse operation (millisecond range) [8]. In the meantime, this preprototype has been modified for longer-pulse operation at $2 \mathrm{MW}$ output power with pulse lengths up to $100 \mathrm{~ms}$ as the first step, and towards $1 \mathrm{~s}$ at a later second step. The modifications focussed primarily on introducing an advanced cooling system [9]. In parallel, a new inverse magnetron injection gun (IMIG) is also ready for testing [10]. The design parameters for the coaxial-cavity pre-prototype are summarized in Table I. The most important technological constraints, which influence the choice of the operating mode and parameters are shown in Table II $[11,12]$. The basic construction of the 2 MW coaxialcavity gyrotron is shown in Fig. 1. As can be seen, the design parameters specify operation at a single frequency of $170 \mathrm{GHz}$.

TABLE I. DESIgn PARAMETERS FOR THE COAXIAL CAVITY Gyrotron [8].

\begin{tabular}{lc}
\hline Parameter & Value \\
\hline Operating cavity mode & $\mathrm{TE}_{34,19}$ \\
Frequency, $f$ & $170 \mathrm{GHz}$ \\
RF output power, $P_{\text {out }}$ & $2 \mathrm{MW}$ \\
Beam current, $I_{B}$ & $75 \mathrm{~A}$ \\
Accelerating voltage, $U_{C}$ & $90 \mathrm{kV}$ \\
Velocity ratio (pitch factor), $\alpha$ & $\sim 1.3$ \\
Cavity magnetic field, $B_{\text {cav }}$ & $6.87 \mathrm{~T}$ \\
Outer cavity radius, $R_{\text {cav }}$ & $29.55 \mathrm{~mm}$ \\
\hline
\end{tabular}

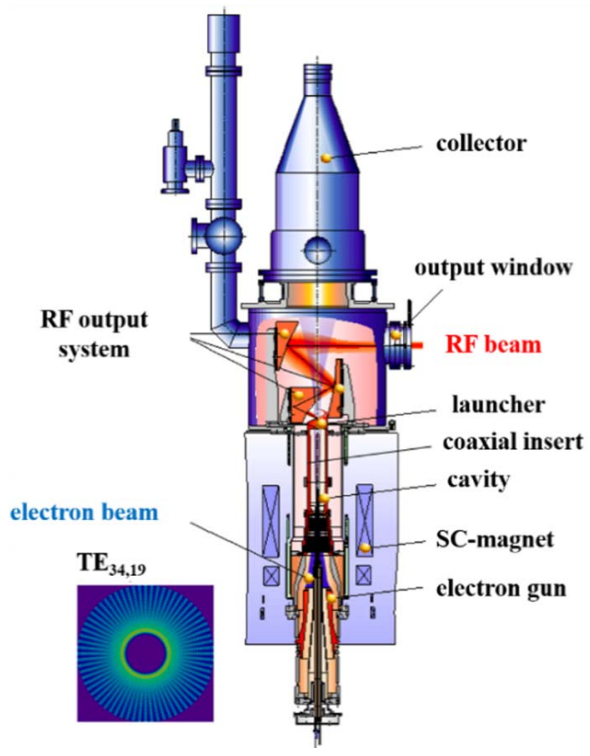

Fig. 1. Sketch of the $2 \mathrm{MW} 170 \mathrm{GHz}$ KIT coaxial-cavity gyrotron [8]. 
TABLE II. TEChNOLOGical Gyrotron DeVelopment Constraints [11], [12].

\begin{tabular}{lc}
\hline Parameter & Value \\
\hline Electric field at emitter & $\leq 7 \mathrm{kV} / \mathrm{mm}$ \\
Emitter current density & $\leq 4 \mathrm{~A} / \mathrm{cm}^{2}$ \\
Outer cavity wall loading & $\leq 2 \mathrm{~kW} / \mathrm{cm}^{2}$ \\
Insert loading & $\leq 0.2 \mathrm{~kW} / \mathrm{cm}^{2}$ \\
\hline
\end{tabular}

However, future DEMO gyrotrons could require operation at doublet, triplet or even quadruplet frequencies formed by 136 $\mathrm{GHz}, 170 \mathrm{GHz}, 204 \mathrm{GHz}$, or $240 \mathrm{GHz}$. The chosen frequencies correspond to the resonances of the output diamond window. This motivated studies shall clarify the feasibility of the existing prototype to be operated at those frequencies whereby the cyclotron frequency is defined by

$$
\Omega_{c} \approx 2 \pi \cdot \frac{28 \cdot B[\mathrm{~T}]}{\gamma}[\mathrm{GHz}],
$$

where $B$ describes the magnetic field and $\gamma$ the relativistic factor which is defined with the beam voltage $U_{\text {beam }}$ by $\gamma=1+$ $U_{\text {beam }} / 511$ [keV].

The operating $\mathrm{TE}_{\mathrm{mp}}$-mode in gyrotrons which are capable for output powers in the MW range has to be volumetric. The definition of volume modes is given in [13] by the condition of an azimuthal mode index of $m>>1$ and transversal mode index

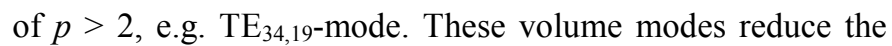
cavity loading and could be developed for smaller beam radii, which could lead to a more compact gyrotron design and thus, a reduced warm borehole diameter. At KIT a suitable extreme volume mode is calculated for the given IMIG [10] in terms of a beam radius of below $8 \mathrm{~mm}$.

This paper is structured in the following way: Section II introduces the selection of the modes for multi-frequency operation and presents the first simulation results. A slight modification of the cavity is performed to improve the output power at higher frequencies. Section III summarizes the results assuming an extreme volume-mode as new operating mode. A comparison of these two approaches including the ongoing investigations are presented in Section IV.

\section{FEASIBILITy StUdy FOR MULTI-FREQUENCY OPERATION}

The $170 \mathrm{GHz}, 2 \mathrm{MW}$ coaxial-cavity design is studied regarding the multi-frequency operation. A possible operation at $170 \mathrm{GHz}, 204 \mathrm{GHz}$ and $238 \mathrm{GHz}$ (window resonances) is targeted. The aim of the study is to check if the actual working gyrotron can operate at the above mentioned frequencies without huge efforts in redesigning of new components. The condition for mode selection is given by a maximal deviation of $<3.6 \%$ in caustic radius compared to the one of the $\mathrm{TE}_{34,19-}$ mode. This is the condition that the quasi-optical system can perform equally well at the different frequencies. The chosen operating modes are determined by the nominal $\mathrm{TE}_{34,19}$-mode at $170 \mathrm{GHz}, \mathrm{TE}_{40,23}-$ mode at $204 \mathrm{GHz}, \mathrm{TE}_{48,26}$-mode at $237 \mathrm{GHz}$, and $\mathrm{TE}_{46,27}$-mode at $238 \mathrm{GHz}$.

Raising the frequency leads to a stronger mode competition due to a denser mode spectrum, which is exemplarily shown for

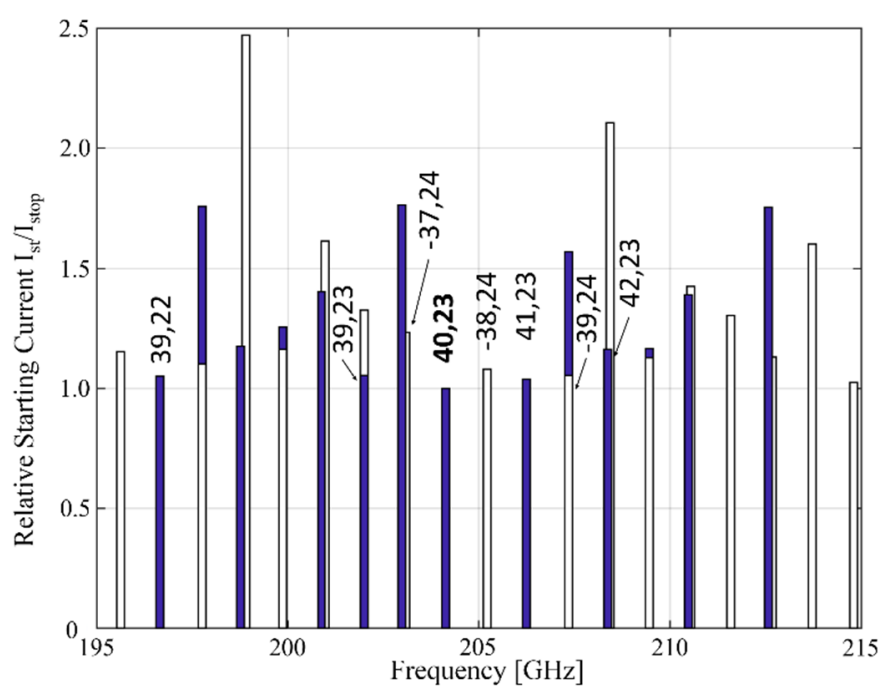

Fig. 2. Minimum starting current, normalized to the operating, co-rotating $\mathrm{TE}_{40,23}$ mode (filled bars: co-rotating modes; empty bars: counter-rotating modes).

the $\mathrm{TE}_{40,23}$-mode in Fig. 2. The nominal mode shows the lowest relative starting current. The most critical or competing modes are those having similar starting currents.

Simulation of the realistic behavior are performed using EURIDICE [14] considering a kinetic energy spread $\Delta E_{\text {kin }}=0.2 \%$ and using the realistic magnetic field of the existing superconductive magnet. Further, the simulations are made with a realistic alpha spread of $\Delta \alpha=20 \% \mathrm{rms}$ due to practical limitations of the magnetron injection gun (MIG) [15]. A velocity ratio $\alpha=v_{\perp} / v_{\|}$of $\alpha=1.2$ instead of $\alpha=1.3$ was used to reduce the risk of unwanted parasitic oscillations, especially at frequencies above $200 \mathrm{GHz}$. EURIDICE offers the opportunity to simulate start-up scenarios while raising the accelerating voltage in a linear way. As an example, in Fig. 3 the adiabatic start-up scenario is shown for the $\mathrm{TE}_{40,23}$-mode. This graph is a typical scenario by raising the beam voltage. First the TE41,23-mode with an almost similar starting current compared to the nominal mode is excited and operates at 206.2 $\mathrm{GHz}$ in respect to Eq. (1). At $65 \mathrm{kV}$ mode switching takes place and the nominal $\mathrm{TE}_{40,23}$ is excited till $73 \mathrm{kV}$. Table III shows the proposed operation parameters and the results of the multimode simulations. The output power at $204 \mathrm{GHz}$ reaches 1.8 MW and is limited by the cavity loading constraint of Table II.

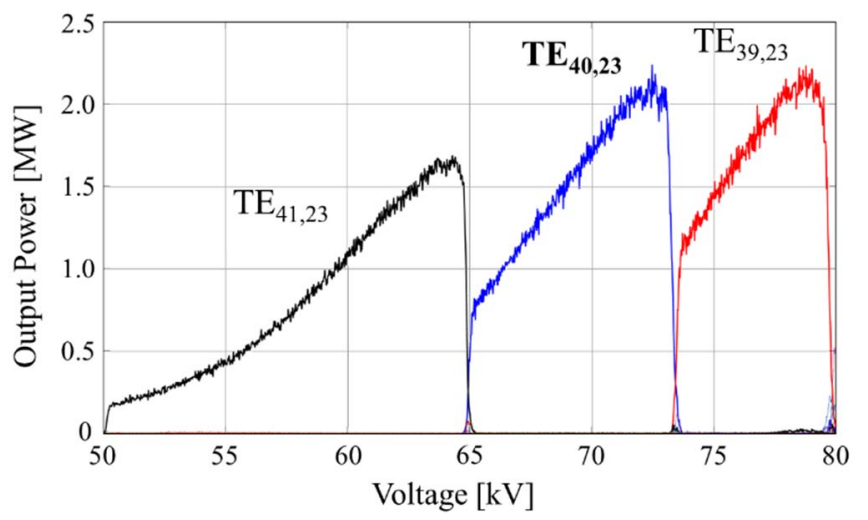

Fig. 3. Start-up Scenario for the existing coaxial-cavity operating on the mode $\mathrm{TE}_{40,23}$ at $204 \mathrm{GHz}$. 
TABLE III. Operation Parameters and Simulation Results of the MULTI-FREQUENCY SIMULATION BASED ON THE EXISTING CAVITY.

\begin{tabular}{|c|c|c|c|c|}
\hline Frequency & 170 & 204.1 & 237.2 & 238.3 \\
\hline Mode & $\mathrm{TE}_{34,19}$ & $\mathrm{TE}_{40,23}$ & $\mathrm{TE}_{48,26}$ & $\mathrm{TE}_{46,27}$ \\
\hline Eigenvalue, $\chi$ & 105.2 & 126.3 & 146.8 & 147.5 \\
\hline Relative caustic radius & 0.3232 & 0.3167 & 0.3270 & 0.3119 \\
\hline Beam voltage [keV] & 90 & 70.5 & 60 & 60 \\
\hline Beam current $[\mathrm{A}]$ & 75 & 80 & 60 & 60 \\
\hline Magnetic field [T] & 6.86 & 8.02 & 9.20 & 9.24 \\
\hline Velocity ratio, $\alpha$ & 1.2 & 1.2 & 1.2 & 1.2 \\
\hline Interaction length [mm] & 16 & 16 & 16 & 16 \\
\hline Beam radius, $R_{\text {beam }}[\mathrm{mm}]$ & 10.0 & 9.77 & 10.11 & 9.65 \\
\hline Quality factor, $Q_{\text {diff }}$ & 2132 & 2955 & 3889 & 4197 \\
\hline Wall loading $\left[\mathrm{kW} / \mathrm{cm}^{2}\right]$ & 1.99 & 1.99 & 1.53 & 1.50 \\
\hline Insert loading [kW/cm²] & 0.11 & 0.06 & 0.002 & 0.002 \\
\hline Output power [MW] & 2.3 & 1.8 & 1.02 & 1.02 \\
\hline Efficiency [\%] & 35.2 & 33.8 & 30.2 & 30.0 \\
\hline
\end{tabular}

The output power at $237 / 238 \mathrm{GHz}$ reach $1.02 \mathrm{MW}$ and is limited by mode stability. The mode series $\mathrm{TE}_{34,19}, \mathrm{TE}_{40,23}$, and $\mathrm{TE}_{48,26}$ offer nearly identical relative caustic radii which implies the opportunity for the usage of the same quasi optical system.

In terms of multi-frequency operation, a comparatively equal output power at each frequency is desired. To this goal, the first modification is done by changing the length of the cavity in order to reduce the quality factor. The midsection length of the cavity is reduced from $16 \mathrm{~mm}$ (i.e. $9 \lambda_{0}$ at $170 \mathrm{GHz}$ ) to $13.6 \mathrm{~mm}\left(\approx 9 \lambda_{0}\right.$ at $\left.204 \mathrm{GHz}\right)$. The insert loading constraint of $0.2 \mathrm{~kW} / \mathrm{cm}^{2}$ is exceeded by decreasing the interaction length further. The shorter cavity results in a decrease in cavity loading and therefore the nominal operation parameters can be adjusted for an increased output power to $2.05 \mathrm{MW}$ at $204 \mathrm{GHz}$. In general, the water cooling of the KIT gyrotron prototype is expected to be further improved in the future and the constraint of the cavity loading could be relaxed. Simulations show that, the maximum stable output power at $204 \mathrm{GHz}$ could be increased from $2.05 \mathrm{MW}$ to approximately $2.35 \mathrm{MW}$ at a cavity loading of $2.1 \mathrm{~kW} / \mathrm{cm}^{2}$ and $34.7 \%$ efficiency. Further, the output power of the $\mathrm{TE}_{34,19}$-mode is decreased to $1.98 \mathrm{MW}$ by shortening the cavity length and no change in the nominal operation parameters, which results in similar output powers at both frequencies. In Table IV the maximum achievable parameters are summarized in the brackets. In the next step the cavity will be modified further increasing the efficiency up to $35 \%$.

TABle IV. Operation Parameters and Simulation Results of the SLIGHTLY MODIFIED CAVITY DESIGN.

\begin{tabular}{lccc}
\hline Mode & $\mathrm{TE}_{34,19}$ & $\mathrm{TE}_{40,23}$ & $\mathrm{TE}_{48,26}$ \\
\hline Beam voltage [kV] & $90(93)$ & $80(82)$ & 78 \\
Beam current [A] & $75(90)$ & 85 & 70 \\
Magnetic field [B] & $6.88(6.89)$ & 8.15 & 9.45 \\
Velocity ratio, $\alpha$ & 1.2 & 1.2 & 1.1 \\
Interaction length [mm] & 13.6 & 13.6 & 13.6 \\
Cavity loading [kW/cm $\left.{ }^{2}\right]$ & $1.5(2.0)$ & $2.00(2.1)$ & 1.85 \\
Insert Loading [kW/cm $\left.{ }^{2}\right]$ & $0.09(0.12)$ & $0.06(0.07)$ & 0.003 \\
Output power [MW] & $1.98(2.78)$ & $2.05(2.35)$ & 1.6 \\
Efficiency [\%] & $30.3(33.9)$ & $31.4(34.7)$ & 30.4 \\
\hline
\end{tabular}

\section{FEASIBILITY OF OPERATION IN AN ULTRA-VOLUME MODE}

Operation of gyrotrons with volume modes (i.e. modes with relative caustic radius $R_{c} / R_{\text {cav }}<0.5$ ) is attractive because such modes have reduced ohmic loading of the cavity and require a smaller beam radius, which could lead to a more compact design in terms of the diameter of the electron gun. The coaxial concept favors the use of volume modes, since it reduces the associated voltage depression. The $\mathrm{TE}_{34,19}$-mode is already a volume mode $\left(R_{c} / R_{\text {cav }}=0.32\right)$. However, for the reasons mentioned above, the possibility of operation with an even more extreme volume mode is being investigated. Given that the coaxial insert cannot be infinitely small (for reasons related to robustness and cooling), a lower limit about $7.5 \mathrm{~mm}$ was considered for the beam radius. This triggered the search for possible operating modes with a relative caustic radius of the order of 0.25 . In particular, based on an optimized design of an IMIG [10] a beam radius of $7.42 \mathrm{~mm}$ is targeted. The azimuthal mode index $m=25$ has been found appropriate at the operating frequency of $170 \mathrm{GHz}$ by using the known formula $\chi_{m-1,1}=$ $2 \pi f R_{\text {beam }} / c$. The modes $\mathrm{TE}_{25,22}, \mathrm{TE}_{25,23}$ and $\mathrm{TE}_{25,24}$ showed suitable output powers, while having similar eigenvalues compared to the nominal $\mathrm{TE}_{34,19}$-mode. The corresponding cavity radii are obtained through the formula $R_{\text {out }}=$ $\chi_{m, p} R_{\text {beam }} / \chi_{m-1,1}$, where the eigenvalue $\chi_{m, p}$ is defined by the $p^{\text {th }}$ root of the characteristic equation [5]. Since all considered $\mathrm{TE}_{25, \mathrm{p}}$-modes showed almost similar output power, the $\mathrm{TE}_{25,22}$ has been selected, due to its lowest eigenvalue $\chi_{25,22}=102.9$ (relative caustic radius 0.245 ) and thus the best potential for mode stability. This mode offers a reduced eigenvalue compared to the eigenvalue of the $\mathrm{TE}_{34,19}$-mode $\left(\chi_{34,19}=\right.$ 105.2). The usage of this mode results in changes of the gyrotron design, compared to the nominal mode. The smaller beam radius allowed by this mode reduces also the cavity diameter. In addition, the higher normalized field amplitude $F$ of this ultra-volume mode requires a shorter cavity length to keep an equally high transverse efficiency $\eta_{\perp}[16]$. The shorter cavity has a smaller diffractive quality factor, which further reduces the ohmic loading. The interaction length amounts to $14.4 \mathrm{~mm}$ and the outer radius is determined to $28.9 \mathrm{~mm}$. On the other hand, care should be taken in order for the loading of the coaxial insert to remain within acceptable levels, given the smaller caustic radius of the mode.

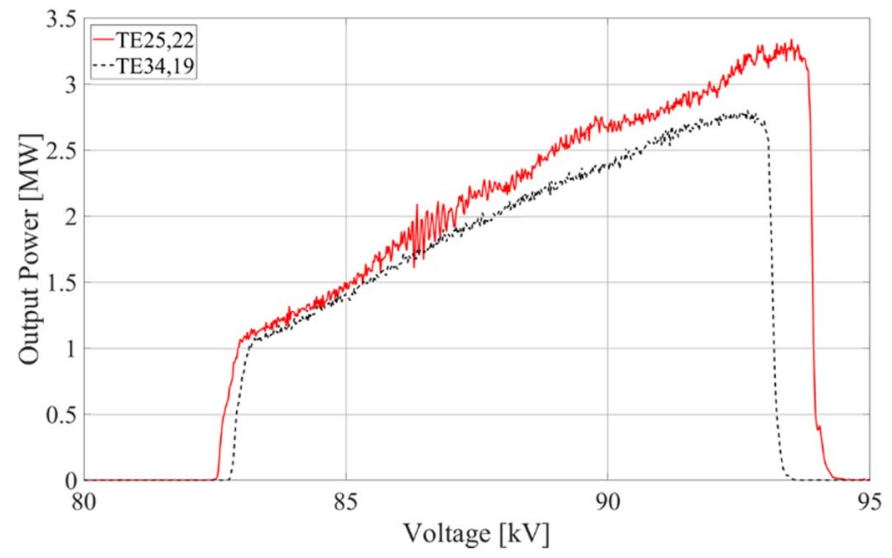

Fig. 4. Comparison of the output power of the modes $\mathrm{TE}_{25,22}$ and $\mathrm{TE}_{34,19}$. 


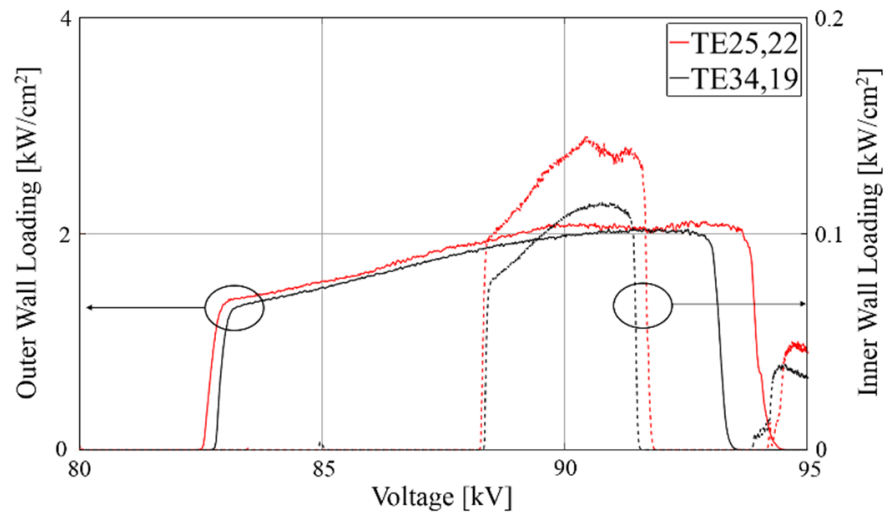

Fig. 5. Comparison of insert and cavity loading between the modes $\mathrm{TE}_{25,22}$ and $\mathrm{TE}_{34,19}$ (solid lines indicate the cavity loading and the dashed lines indicate the insert loading).

Numerical simulations with EURIDICE show that the $\mathrm{TE}_{25,22}$-mode can operate at higher beam currents $(87.5 \mathrm{~A})$ then the nominal mode $\mathrm{TE}_{34,19}(75 \mathrm{~A})$, while still satisfying the maximum wall load constraint. This enables the $\mathrm{TE}_{25,22}$ to produce about $30 \%$ higher output power in comparison to the nominal $\mathrm{TE}_{34,19}$-mode, as depicted in Fig. 4. The extremely high beam current (up to $90 \mathrm{~A}$ ) is located in the range of the possibility with the new KIT IMIG. Figure 5 shows the corresponding ohmic loading of these two modes. The constraints of maximum cavity loading of $2.0 \mathrm{~kW} / \mathrm{cm}^{2}$ and insert loading of $0.2 \mathrm{~kW} / \mathrm{cm}^{2}$ are still maintained. In general, the choice of suitable dimensions for the inner rod is mandatory, due to its strong effect on the insert loading. The radius of the inner conductor is reduced to $5.57 \mathrm{~mm}$ instead of $7.5 \mathrm{~mm}$. Therefore, thermomechanical simulations are necessary for evaluating the existing cooling system of the inner conductor.

\section{SUMMARY AND OUTLOOK}

In this presentation, it is shown by simulation that the

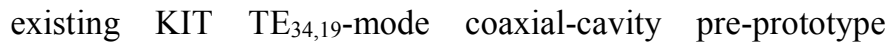
gyrotron is capable of operation as a multi-purpose/multifrequency device. At the frequency of 170 (204) GHz, an output power of 2.3 (1.8) MW can be achieved. A small modification of the cavity would allow an output power of up to 2.78 (2.35) MW even. In next steps the cavity will be optimized regarding a higher interaction efficiency and output power. Secondly, the selection of a new extreme volume-mode as main operating mode is discussed for reducing the overall gyrotron diameter and further increased power at $170 \mathrm{GHz}$. This mode shows an increase in output power of approximately $30 \%$ compared to the nominal $\mathrm{TE}_{34,19}$-mode. Besides the increased output power up to $3.2 \mathrm{MW}$, the reduced beam radius (typically $7.42 \mathrm{~mm}$ ) could lead to a more compact gyrotron design. This new volume mode will be studied further towards $170 / 204 \mathrm{GHz}$ multi-frequency operation. In the next step the simulations will become even more realistic by considering both the cavity and the non-linear up-taper as well as the precise electron beam parameters of the existing gun, as obtained via the code ARIADNE [17].

\section{ACKNOWLEDGMENT}

This work has been carried out partly within the framework of the EUROfusion Consortium and has received funding from the Euratom research and training program 2014-2018 under grant agreement No. 633053. The views and opinions expressed herein do not necessarily reflect those of the European commission. Parts of the simulations presented in this work have been carried out the Marconi-Fusion super-computer facility.

\section{REFERENCES}

[1] H. Braune, et al., "Gyrotron operation during the first W7-X campaign handling and reliability, " in Proc. IRMMW, Copenhagen, Denmark, Sep. 2016, pp. 1-2.

[2] T. Omori, et al., "Overview of the ITER EC H\&CD system and its capabilities," Fusion Eng. Des., vol. 86, pp. 951-954, Mar. 2011.

[3] G. Federici, et al., "EU DEMO design and R\&D studies," in Proc. $25^{\text {th }}$ SOFE, San Francisco, CA, USA, Jun. 2013, pp. 1-8, paper WO1-1.

[4] Z. C. Ioannidis et al., "CW experiments with the EU 1-MW, 170-GHz industrial prototype gyrotron for ITER at KIT," IEEE Trans. on Electron Devices, vol. 64, no. 9, pp. 3885-3892, Sept. 2017.

[5] T. Rzesnicki, et al., "Experimental verification of the european $1 \mathrm{MW}$, $170 \mathrm{GHz}$ industrial CW prototype gyrotron for ITER," Fusion Eng. Des. (2017), http://dx.doi.org/10.1016/j.fusengdes.2017.02.021.

[6] C. T. Iatrou, et al., "Coaxial cavities with corrugated inner conductor for gyrotrons," IEEE Trans. Microwave Theory Tech., vol. 44, pp. 56-64, 1996.

[7] K. A. Avramides, et al., "Design considerations for powerful continuouswave second-cyclotron-harmonic coaxial-cavity gyrotrons," IEEE Trans. on Plasma Science, vol. 32, no. 3, 2004.

[8] T. Rzesnicki, et al., "2.2-MW record power of the 170-GHz european preprototype coaxial-cavity gyrotron for ITER," IEEE Transactions on Plasma Science, Vol. 38, No. 6, pp. 1141-1149, 2010.

[9] J. Jelonnek, et al., "Design consideration for future DEMO gyrotrons: A review on related gyrotron activities within EUROfusion," Fusion Eng. Des. (2017), http://dx.doi.org/10.1016/j.fusengdes.2017.01.047.

[10] S. Ruess, et al., "An inverse magnetron injection gun for the KIT 2-MW coaxial-cavity gyrotron," IEEE Trans. on Electron Devices, vol. 63, no. $5,2016$.

[11] K. A. Avramides, C. T. Iatrou, and J. L. Vomvoridis, "Systematic procedure for operating-mode selection in conventional and coaxialcavity gyrotrons", EC-14, 14th Joint Workshop on Electron Cyclotron Emission and Electron Cyclotron Resonance Heating, 9-12 May 2006, Santorini, Greece, Conference Proceedings, pp. 484-489, A. Lazaros Ed., http://www.hellasfusion.gr.

[12] P. C. Kalaria, et al., "Systematic cavity design approach for a multifrequency gyrotrons for DEMO and study of its RF behaviour," Physics of Plasma, 23(9), 2016.

[13] G. Gantenbein, et al., "Design if a high order volume mode cavity for a 1 MW/140 GHz gyrotron," Int. Journal of Electronics, 78:4, 771-787, DOI:10.1080/00207219508926209.

[14] K. A. Avramides, et al., "EURIDICE: A code-package for gyrotron interaction simulations and cavity design”, EC-17, 17th Joint Workshop on Electron Cyclotron Emission and Electron Cyclotron Resonance Heating, 7-11 May 2012, Deurne, The Netherlands, EPJ Web of Conferences 32, 04016 (2012).

[15] J. M. Baird and W. Lawson, "Magnetron injection gun (MIG) design for gyrotron applications," International Journal of Electronics, vol. 61, no. 6, pp. 953-967, 1986

[16] B. G. Danly and R. J. Temkin, "Generalized nonlinear harmonic gyrotron theory," The Physics of Fluids 29, 561, 1986.

[17] I. Gr. Pagonakis and J. L. Vomvoridis. "The Self-Consistent 3D Trajectory Electrostatic Code ARIADNE for Gyrotron Beam Tunnel Simulation," Infrared and Millimeter Waves, Conference Digest of the 2004 Joint 29th International Conference on 2004 and 12th International Conference on Terahertz Electronics, 2004., 2004, pp. 657-658. 